\title{
Lipid-raft phase modulation by membrane-anchored proteins with inherent phase separation properties
}

Il-Hyung Lee ${ }^{1 *}$, Matthew Y. Imanaka ${ }^{2}$, Emmi H. Modahl ${ }^{1}$, Ana P. Torres-Ocampo ${ }^{3}$

${ }^{1}$ Department of Chemistry, University of Puget Sound, Tacoma, WA 98416, USA; ${ }^{2}$ Department of Biology, University of Puget Sound, Tacoma, WA 98416, USA; ${ }^{3}$ Department of Biochemistry and Molecular Biology, University of Massachusetts Amherst, Amherst, MA 01003, USA

The file includes:

Figs. S1 to S4

Supporting Methods 


\section{Supporting Figures}

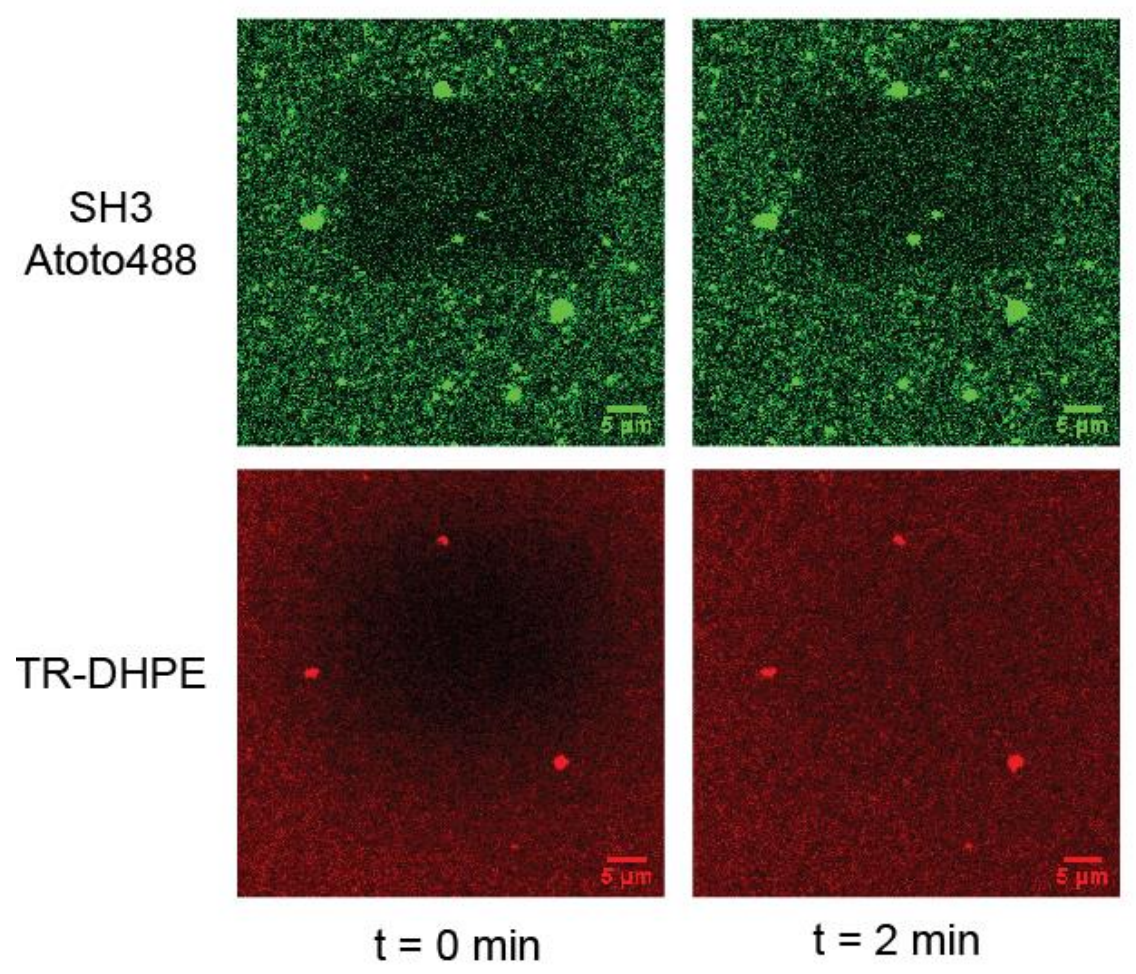

Figure S1. Fluorescence recovery after photobleaching (FRAP) shows partially mobile lipid membrane after sequential incubation of SH3x4 and PRMx4 on supported lipid bilayer. (SLB) SLB composition was $98 \%$ DOPC, $2 \%$ Ni-NTA and $0.01 \%$ TR-DHPE. $2 \mu \mathrm{M} \mathrm{SH} 3 \times 4$ with polyhistidine tag was incubated for $30 \mathrm{~min}$ and washed with buffer. $2 \mu \mathrm{M}$ of PRMx4 was introduced and incubated for $30 \mathrm{~min}$. After the incubation FRAP was performed to check fluidity of the membrane. Protein molecules are only partially mobile, and unlinked lipid molecules are mostly fluidic shown by full FRAP recovery in 2 min. Small clusters were also visible on the biliayer which may have formed due to favorable multivalent binding between $\mathrm{SH} 3 \times 4$ and PRMx4. Scale bars are $5 \mu \mathrm{m}$. 
a

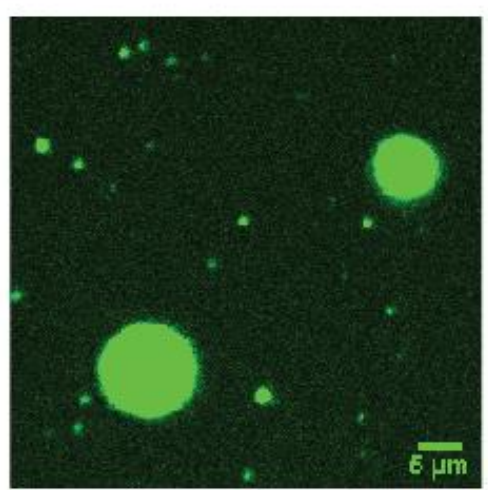

b
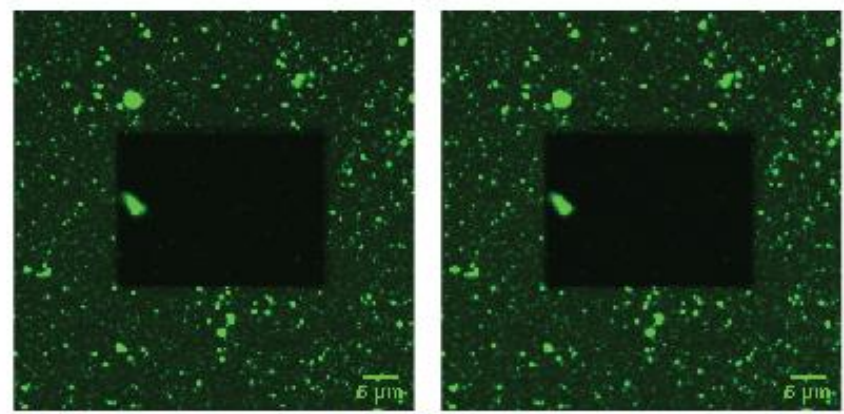

DDX4

-GFP

\section{TR-DHPE}

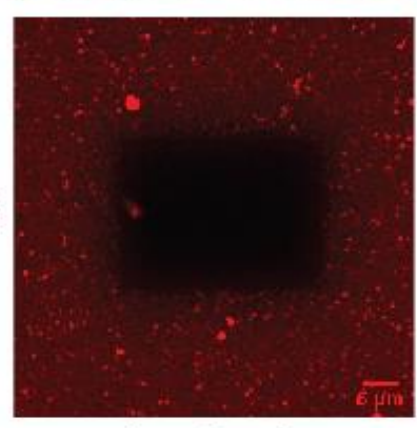

$\mathrm{t}=0 \mathrm{~min}$

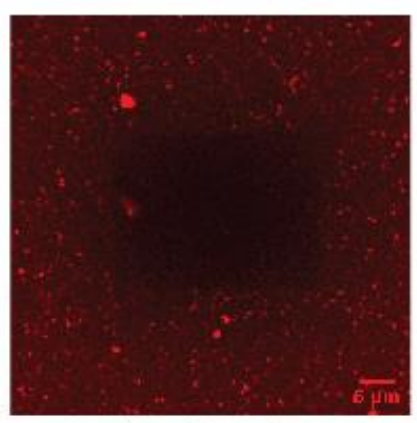

$t=2 \min$

Figure S2. DDX4-GFP forming 3D droplets in solution and immobile lipid membrane on SLB (a) An example image of solution phase separated domains or droplets formed by DDX-GFP at high concentration $>100 \mu \mathrm{M}$ (b) FRAP recovery of SLB after 30 min incubation with $1 \mu \mathrm{M}$ for 30 min. SLB composition was $90 \%$ DOPC, $10 \% \mathrm{Ni}-\mathrm{NTA}$ and $0.01 \%$ TR-DHPE. DDX4-GFP showed almost no recovery and TR-DHPE channel recovered partially. Small clusters were also visible on the biliayer which may have formed due to favorable interaction of DDX4-GFP. Scale bars are $5 \mu \mathrm{m}$. 


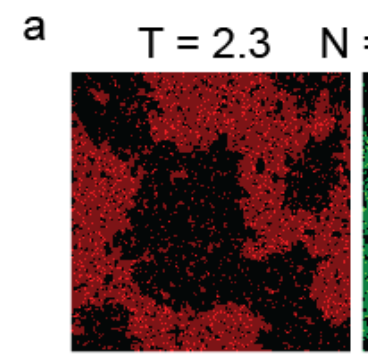

Lower

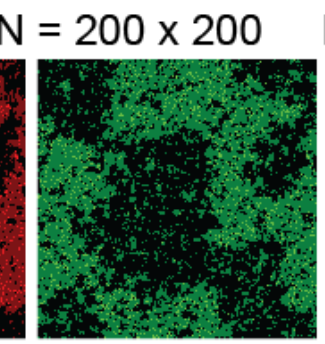

Upper

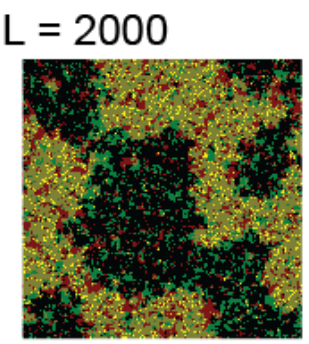

Merged

b

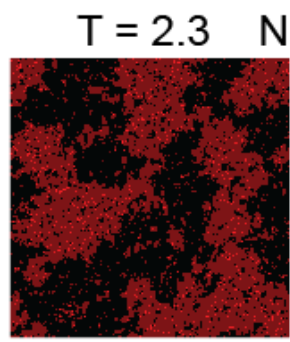

Lower

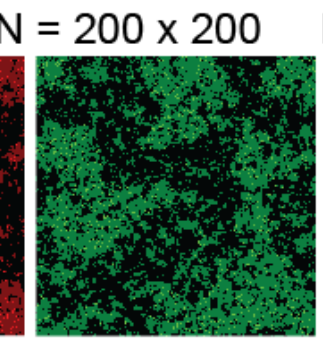

Upper
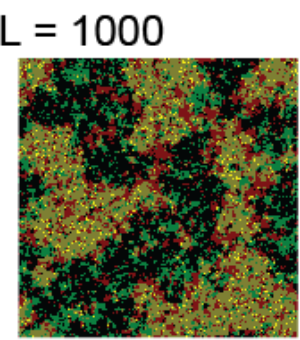

Merged

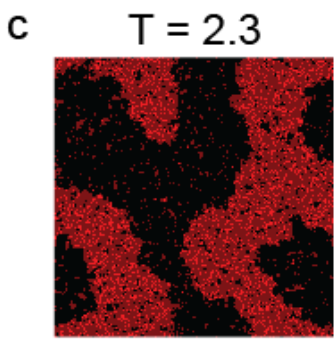

Lower

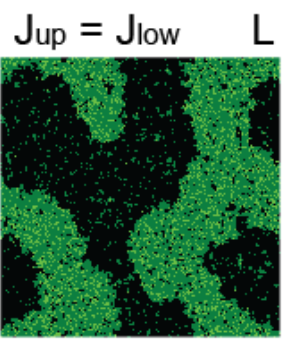

Upper
$L=4000$

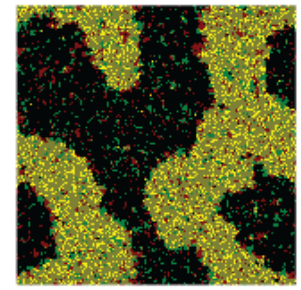

Merged

d

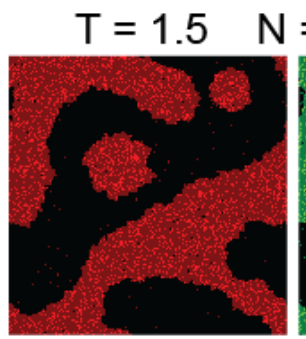

Lower

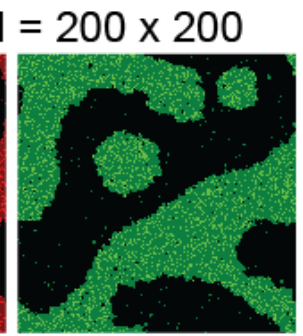

Upper

$\mathrm{L}=4000$

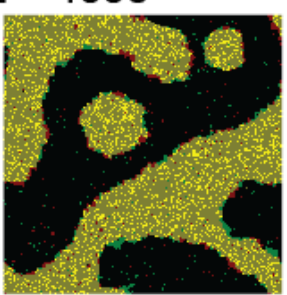

Merged

Figure S3. Additional Ising model simulation data show more coupling between two layers generates more enhanced and correlated phase separation. Spin states of a simulation after $\mathrm{t}=$ 20,000 steps. For lower layer, black and red colors indicate two spins. For upper layer, black and green colors indicate two spins. $\mathrm{T}=2.3$ was the critical temperature for the lower layer and the upper layer was above its critical temperature due to lower inter-particle stabilization energy (0.9 of the lower layer) for all simulations except $\mathrm{c}$ where both layers had equal stabilization energy $\mathrm{J}$. $\mathrm{N}$, or the total number of spins were 40,000 for each layer. (a) Spin states for $\mathrm{T}=2.3, \mathrm{~L}=2000$. $5 \%$ of spins were linker spins and the system still showed strong correlation and enhanced phase separation. (b) Spin states for $\mathrm{T}=2.3, \mathrm{~L}=1000.2 .5 \%$ of spins were linker spins and the system still showed strong correlation and enhanced phase separation. As L lowers, we saw less dense phase segregation. (c) Spin states for $\mathrm{T}=2.3, \mathrm{~L}=4000$ with equal energetic stabilization $\mathrm{J}$ for 
both layers. Correlated movement and enhanced phase separation could be observed. As $J_{\text {upper }}$ is higher than other simulations (1.0 instead of $0.9 \mathrm{~J}_{\text {lower }}$ ), we could see further enhanced phase separation behavior (d) Spin states for $\mathrm{T}=1.5, \mathrm{~L}=4000$. At temperature far below the critical temperature, denser phase separation was observed as expected and two layers were very strongly correlated.

a

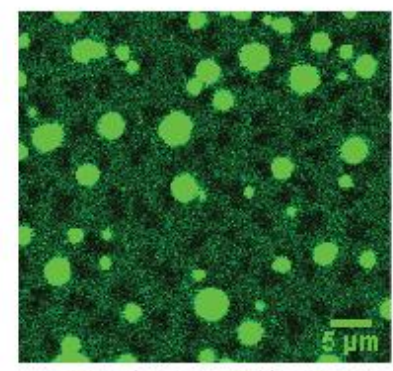

Peptide-Atto488

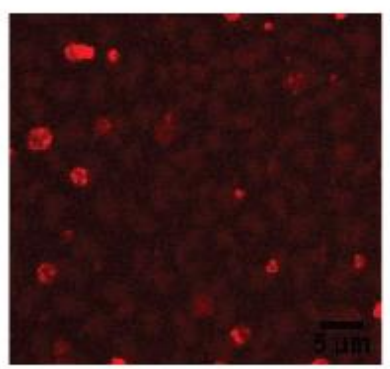

TR-DHPE

b

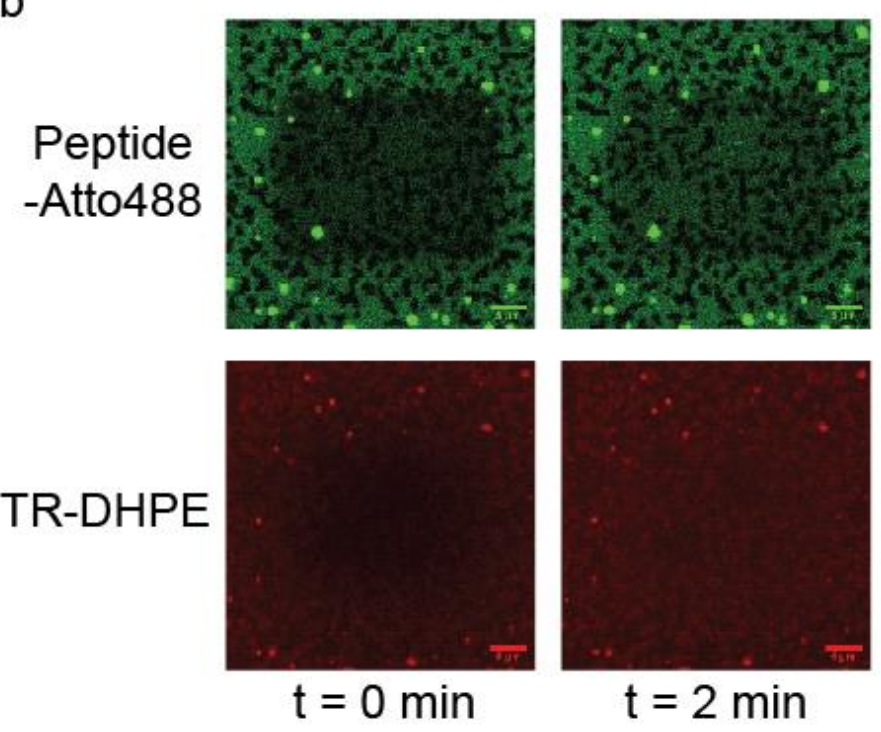

Figure S4. Three-component protein system on the supported lipid bilayer. (a) Three component system forms a network of protein layer on the membrane, and 3D phase droplets tend to smear on the membrane forming circular domains which were at least an order of magnitude concentrated than proteins on the membrane. (b) Fluorescence recovery after photobleaching (FRAP) shows pretty mobile lipid membrane after the incubation. Protein channel recovered > $50 \%$ and lipid channel recovered nearly $100 \%$ within 2 min after photobleaching. SLB composition was 98\% DOPC, 2\% Ni-NTA and 0.01\% TR-DHPE. All three proteins were originally mixed as $25 \mu \mathrm{M}$ each in buffer which readily formed phase droplets. The solution was then diluted into $5 \mu \mathrm{M}$ to interact with the SLB. The images were taken after 30 min of incubation. 


\section{Supporting Methods}

\section{DDX4-GFP gene sequence}

The yellow colored region is from the IDR of DDX4 and the green colored region is for GFP

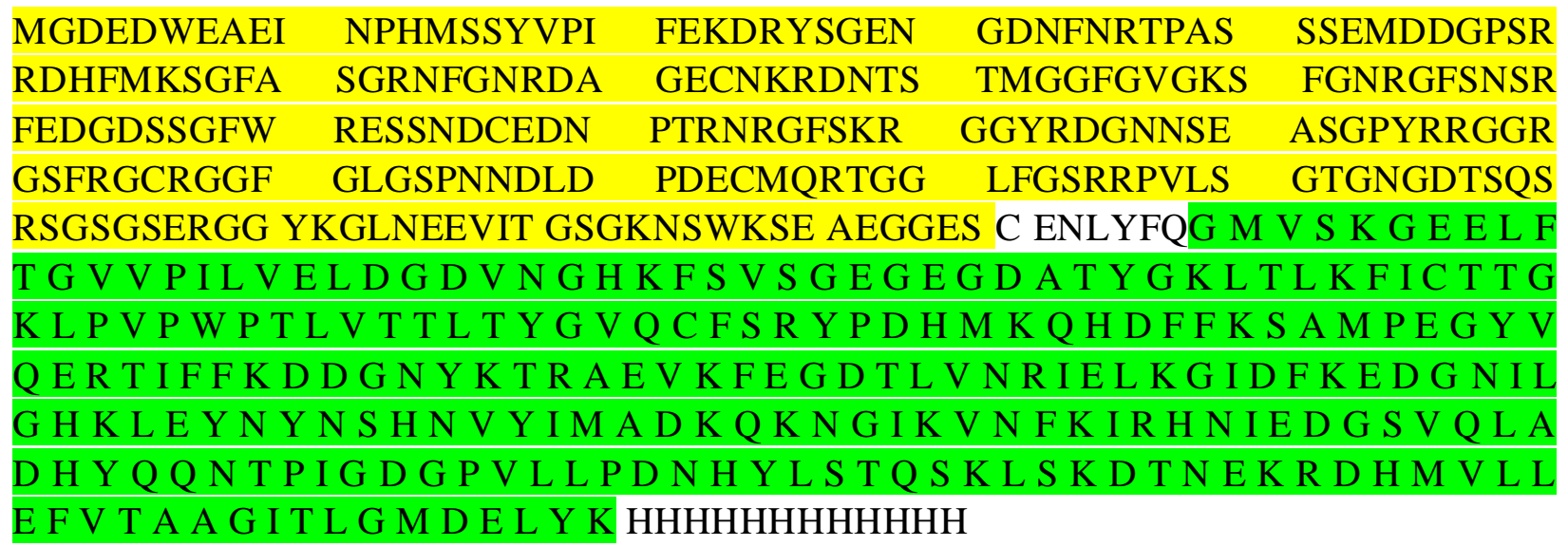

\section{$\underline{\text { LAT sequence }}$}

HHHHHHHHHHGGSGGSGGD \{pY\}VNVGGSGGSGGD \{pY $\}$ VNVDKKC

\section{$\underline{\text { SLB protocol }}$}

Small unilamellar vesicles (SUV) were made by extrusion. Net $2 \mathrm{mg}$ of lipid mixture in chloroform solution was dried on a clean round bottom flask surface by $30-45$ min of rotavap drying at $50{ }^{\circ} \mathrm{C}$. The dried lipid film was further dried by $2-5 \mathrm{~min}$ of Nitrogen blow. $1 \mathrm{ml}$ of Milli-Q water was added to perform three times of freeze thawing. The sample was completely frozen by lowering temperature of the sample by dry ice then was thawed by raising the temperature in water bath. Completely hydrated lipid solution was then extruded nine times through $100 \mathrm{~nm}$ pore extrusion sheet. Nanosizer extrusion kit from T\&T scientific was used. Collected SUV solution was kept at $4{ }^{\circ} \mathrm{C}$ to use within a week.

To prepare SLB, round cover glasses (Fischer Scientific) were cleaned by bath sonication for 30 min in isopropyl alcohol: $\mathrm{H}_{2} \mathrm{O}=1: 1 \mathrm{v} / \mathrm{v}$ solution. After multiple rinsing, the cover glasses were piranha etched for $2-5 \mathrm{~min}$ in acid piranha solution. (Sulfuric acid: $\mathrm{H}_{2} \mathrm{O}_{2}$ solution = 3:1 v/v) The cover glasses were thoroughly rinsed with Milli-Q water and then used within 24 hours. One of the cover glasses was assembled into the home built stailess steel sample chamber unit, and SUV with net $0.4 \mathrm{mg}$ of lipids was incubated on top of the glass in 1x PBS 2-5 mM MgCl 2 . After 45 min incubation for spontaneous SLB formation, the solution above SLB was washed by exchanging buffer $>5$ times without exposing the lipid surface in the air. Optionally, it was further incubated with $100 \mathrm{mM} \mathrm{NiSO}_{4}$ solution for 5 min to completely charge Ni-NTA lipids with $\mathrm{Ni}^{2+}$ ions. 


\section{Ising model simulation}

\section{Initialization}

Two layers of 200 by 200 spins, represented by two 200 by 200 number matrices were initialized by randomly mixing up-spins and down-spins at high temperature. Spins were randomized without changing the total number of up-spins and down-spins in each layer. There were total 40,000 spins in each layer and 20,000 of them were up-spins and another 20,000 of them were down-spins. L of linker-spins were assumed where spins in two layers at the same position ( $\mathrm{x}, \mathrm{y}$ coordinate) were coupled, so the spin could only move by also moving its coupled spin on the other layer. Two coupled spins in two layers were both assumed to be all up-spins. Linker-spin positions were also randomized in the initialization.

2. Spin exchange simulation (a single step)

2a) One spin was chosen randomly.

2b) A random movement was decided randomly. It was one of four possible movements. \pm 1 in $x$ coordinate or \pm 1 in y coordinate. A movement means two spins will exchange positions effectively moving the chosen spin toward that direction. Spins were not allowed to move between two layers. Periodic boundary condition was assumed for both layers.

2c) $\Delta \mathrm{E}$ of the proposed movement was calculated according to the following equation.

$\Delta \mathrm{E}=-\mathrm{J} \times[$ [ (\# of neighboring identical spins after movement $)-(\#$ of neighboring different spins after movement $)\}-\{$ (\# of neighboring identical spins before movement) - (\# of neighboring different spins before movement) $\}]$

$\mathrm{J}=1.0$ for lower layer and $\mathrm{J}=0.9$ for upper layer. By the unitless definition we used in our simulations, it means at $\mathrm{T}=2.3$, the lower layer was at its original critical temperature ( $\mathrm{T}_{\mathrm{C}}$ of the system is 2.3 when $\mathrm{J}=1.0$ assuming a single layer 2D Ising model), but the upper layer was still above its original transition temperature or was at homogeneous temperature. Neighboring spins were only considered in xy coordinates and two spins at the same position of two layers were not considered as neighboring spins. ( $\mathrm{z}$ coordinate neighboring was not considered) This was to assume the experimental situation where proteins and lipids affected each other only through anchors or there was no direct lipid-protein binding.

If the chosen spin was a linker-spin, simultaneous movement of spins at both layers was assumed and the difference in energy was also calculated accordingly.

2d) The movement was accepted or rejected based on $\Delta \mathrm{E}$. When $\Delta \mathrm{E}<0$, the movement was accepted and spins were exchanged. When $\Delta \mathrm{E} \geq 0$, a random number was generated to make a decision. The movement was accepted only when the random number was smaller than the exp($\Delta \mathrm{E} / \mathrm{T})$, and the movement was rejected when it was larger than the probability. It naturally increased the chance of movement at higher temperature. When the movement was rejected, no spin exchange was performed. 
2e) 2 a to $2 \mathrm{~d}$ was repeated $2 \times 40,000$ times for a unit step. A unit step was a minimal unit of a reaction time in simulation.

\section{Continued dynamic simulation}

Step 2 was repeated for desired amount of time to reach an equilibrated and a representative state of the system at the simulated temperature. $t=2,000,000$ steps calculation was performed for each simulation which was enough to reach the representative state of the system. Spin states of both layers were recorded at every 100,000 step to closely monitor the change of states.

\section{Analyzing the result}

After ensuring that the final spin state at $\mathrm{t}=2,000,000$ was a representative state, the final state was used to interpret the result of the simulation. 